Journal of Case Reports 2019;9(2):114-116

\title{
Encephalitis with Anterior Ethmoidal Neuralgia
}

\author{
Xiao Feng \\ Department of ENT, Clinical Medicine College (Huaihe Hospital), Henan University, Kaifeng city, Henan Province 475001, China.
}

\begin{abstract}
Corresponding Author:
Dr. Xiao Feng

Email: fengxiaobaby@126.com

This is an Open Access article distributed under the terms of the Creative Commons Attribution License (creativecommons.org/ licenses/by/3.0).
\end{abstract}

Received : December 31, 2018

Accepted : May 18, 2019

Published : May 30, 2019

\begin{abstract}
Background: Fever, headache and confusion are considered as the cardinal symptoms of encephalitis. The symptoms of encephalitis can mimic as rhinitis, familiarity with the clinical spectrum and features is important for clinicians to optimize clinical management. Case Report: A 30-year-old female patient presented with sinus area tenderness, but otherwise, she appeared healthy and conscious. She was thought of having rhinitis, due to her past history of having colds. However, application of ephedrine into the nasal cavity aggravated her headache. Headache improved significantly following anti-viral treatment and reduction of intracranial pressure. However, ethmoid area tenderness persisted, which was relieved by using tetracaine spraying into the nasal cavity. Conclusion: Considering her headache started at the canthal area, the organism might reach intra-cranial through anterior ethmoidal nerve. The anterior ethmoidal neuralgia might be a post-encephalitis sequel like post-encephalitis epilepsy.
\end{abstract}

Keywords: Encephalitis, Headache, Neuralgia, Nose, Rhinitis, Sinusitis.

\section{Introduction}

The incidence of encephalitis is relatively low [1], especially among the adult population and in non-tropical areas. The main symptoms of encephalitis are headache, fever and confusion. However, headache is common medical complaint with various underlying causes and many patterns of presentation. As different headaches have different characteristics, it is essential to establish an etiology whenever patients with headache are examined. Establishing a correct diagnosis for otolaryngologist and differentiating rhinitis or sinusitis from encephalitis is challenging especially when only headache and canthal area tenderness, is present.

\section{Case Report}

A 30-year-old female patient presented with complaint of headache following an episode of cold. She experienced persistent pain on the bilateral frontal and temporal aspect of head which was not associated with fever, shivering, or vomiting. She denied nasal blockade, liquid snivel or purulent snivel. First, she visited an otolaryngologist, who found no abnormality in her nasal cavity and advised sinus-coronal computed tomography (CT). Results of CT suggested mild turbinate hypertrophy [Fig.1]. The otolaryngologist prescribed ephedrine dropping into the nasal cavity, which failed to relieve her headache. Then, she visited a neurologist, who advised her hospital admission. Her vitals were temperature $36.5^{\circ} \mathrm{C}$; pulse 75 beats $/ \mathrm{min}$; respiration 17 times/min; blood pressure 115/72 $\mathrm{mmHg}$. The lady was well nourished, had normal development, normal olfaction and meningeal signs were absent. Results of blood routine examination found that total leukocyte count was $20 \times 10^{9} / \mathrm{L}$. There was no abnormal signal upon cranial magnetic resonance imaging (MRI) [Fig.2] and cerebrovascular magnetic resonance angiography (MRA). The sinus computer tomography (CT) failed to suggest sinusitis in this patient. The pressure of cerebrospinal fluid (CSF) was $240 \mathrm{~mm} \mathrm{H} \mathrm{H}_{2} \mathrm{O}$ (normal range is 
80-180 $\mathrm{mm} \mathrm{H}_{2} \mathrm{O}$ ). The results of bacterial culture for acid-fast bacillus and Cryptococcus histolyticus were negative. The patient was given mannitol for reducing intracranial pressure and antiviral drug ganciclovir sodium. Following a 10-day treatment, the patient's leukocyte count, intracranial pressure returned to normal and the patient's headache was relieved. However, the ethmoid area tenderness persisted. She went back to the otolaryngologist for additional help. The doctor provided an endoscopic examination of her nasal cavity, which was found to be normal [Fig.3]. During treatment, the patient was under regular follow-up with use of tetracaine spary in nasal cavity. At the end of the treatment, the patient was symptom free, with absence of ethomoid tenderness.

\section{Discussion}

The following inferences are derived based on this case report. First, based on the clinical data and the symptoms, there are little indications to support the diagnosis of encephalitis except for the presence of headache and high intracranial pressure. Second, the location of the headache is front-temporal, which is also the location of rhinogenous headache. Third, all signs of neurological examination were negative, i.e. no fever and vomiting. These three reasons led to the initial diagnosis as rhinitis, especially in an outpatient facility. Our diagnosis of encephalitis was based on epidemiological features, physical and neurological examinations, cerebrospinal fluid evaluation, electroencephalogram (EEG), CT, MRI $[1,2]$. The onset of the headache was in the mid-September following an episode of cold. Results of all physical examinations, EEG, CT, MRI and MRA were normal except for high intracranial pressure. In a non-epidemic setting, the most common viral cause of focal encephalopathic finding is herpes simplex virus [2]. It is worthy to call to the attention that some severe viral infection of encephalitis can have normal CSF [3].

Following the antiviral treatment, the headache was almost relieved, except that the

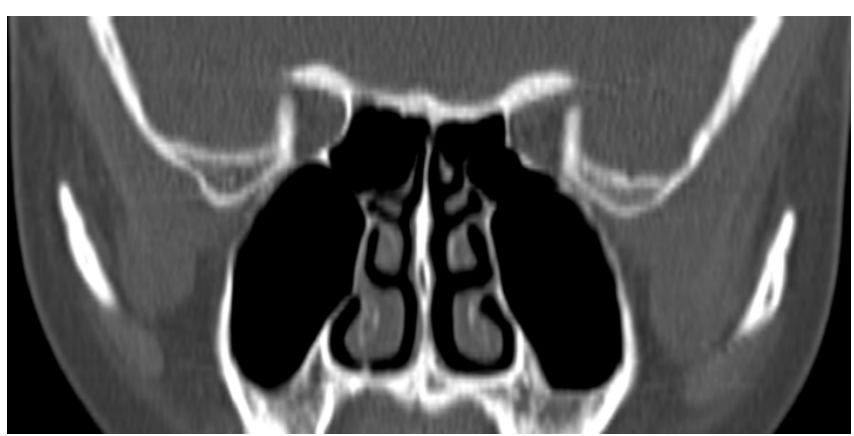

Fig.1: Normal sinus coronal position CT showing absence of inflammation in nasal cavity and sinus.

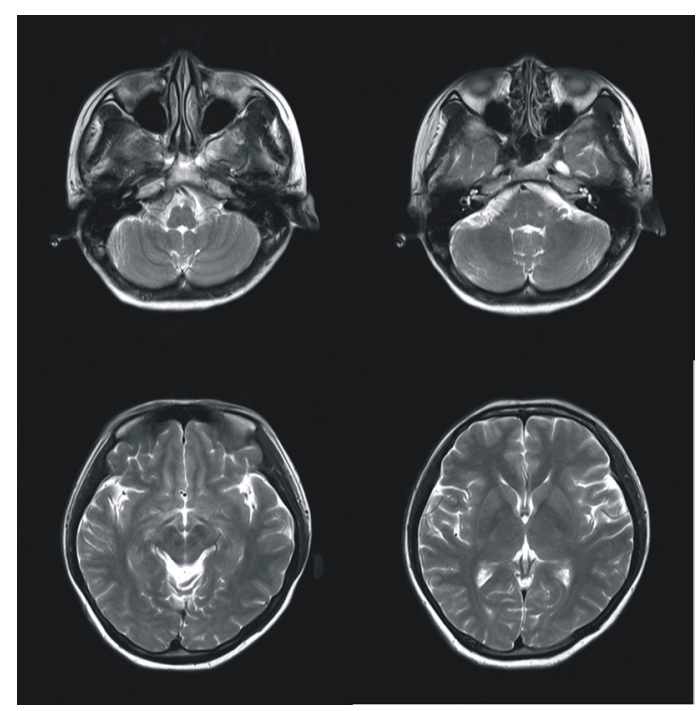

Fig.2: Normal MRI brain.
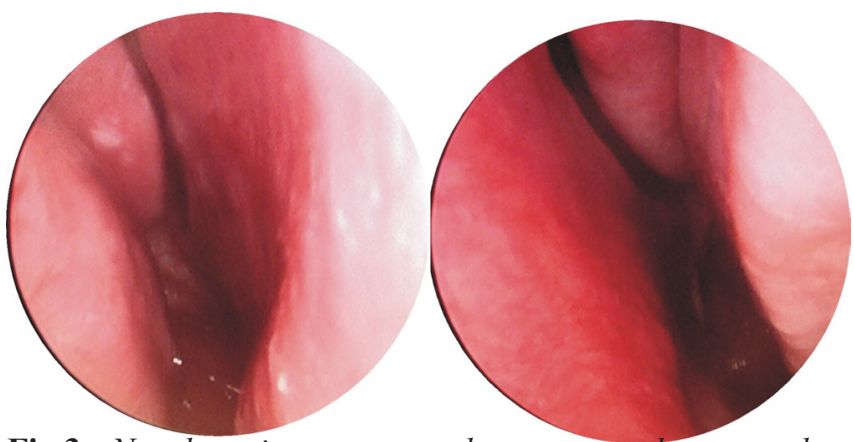

Fig.3: Nasal cavity-no mucosal contact and no purulent snivel.

patient still felt tenderness in the ethmoid area. In order to find the cause of headache following a successful treatment of encephalitis, endoscopic examination of nasal cavity was done to exclude 
mucosal congestion. Headache caused by mucosal congestion [3] can also take place without inflammation in the nasal cavity and the sinus. Headache caused by mucosal congestion is located in the peri-orbital and media canthal or temporozygomatic and is referred pain through the trigeminal system. According to the description from the International Headache Society (IHS), mucosal congestion headache can be abolished within $5 \mathrm{~min}$ following topical application anesthesia to the middle turbinate or contact point area. Anterior ethmoidal neuralgia was considered etiology for headache as her headache was not relieved following the use of ephedrine at the onset of headache and we did not find any contact point in her nasal cavity. Her headache was abolished after using anesthesia, which led us to suspect neuralgia. Considering her headache began at the canthal area, possibility of intracranial involvement through anterior ethmoidal nerve was considered and anterior ethmoidal neuralgia might be a post-encephalitis sequel just like postencephalitis epilepsy. The shortcoming of this paper is that we did not analyze the virus typing of cerebrospinal fluid infection further. However, anti-virus treatment played effect, which indicated the viral encephalitis.

\section{Conclusion}

Encephalitis presenting as headache can be challenging to recognize for an otolaryngologist. Viral encephalitis can initially present as runny nose and nasal obstruction, symptoms which are similar to that of a common cold (acute rhinitis).

Acknowledement: I thank retired Pro. Chung Lee of Northwestern University, for his valuable suggestion and guidance.

Contributors: XF wrote the manuscript and did patient management. He will act as guarantor and approved the final version of this manuscript.

Funding: None; Competing interests: None stated.

\section{References}

1. Steiner I, Budka H, Chaudhuri A, Koskiniemi M, Sainio K, Salonen O. Viral meningoencephalitis: a review of diagnostic methods and guidelines for management. Eur J Neurol. 2010;17(8):957-999.

2. Whitley RJ, Gnann JW. Viral encephalitis: familiar infections and emerging pathogens. Lancet. 2002;359:507-513.

3. Behin F, Behin B, Bigal ME, Lipton RB. Surgical treatment of patients with refractory migraine headaches and intranasal contact points. Cephalalgia. 2005;25(6):439-443. 